\section{Application of Composted Poultry Litter as a Fertilizer for Landscape Bedding Plants}

\author{
S. Christopher Marble ${ }^{1}$, Jeff L. Sibley ${ }^{2,4}$, and Charles H. Gilliam ${ }^{2}$ \\ Auburn University Department of Horticulture, 101 Funchess Hall, Auburn, \\ AL 36849
}

\author{
H. Allen Torbert ${ }^{3}$ \\ USDA-ARS National Soil Dynamics Laboratory, 411 S. Donahue Drive, \\ Auburn, AL 36832
}

Additional index words. landscape annuals, manure, organic fertilizer

\begin{abstract}
Each year, over 16 million tons of poultry litter is produced in the United States. Federal and state regulations now limit the amount of poultry litter that can be land-applied, making it difficult to store and dispose of poultry litter. The objective of this study was to evaluate composted poultry litter (CPL) as a fertilizer source for bedding plants at various rates in comparison with commercially available inorganic fertilizers in regard to plant growth and nutrient leaching. Two experiments were conducted to evaluate use of CPL as fertilizer for landscape annual bedding plants. Petunia spp. 'Celebrity Red' and Verbena hybrida 'Quartz Scarlet' were planted in raised beds simulating an urban landscape. Before planting, 10 inorganic fertilizer or CPL treatments were incorporated into the raised beds, including Peafowl ${ }^{\circledR}$ brand garden-grade fertilizer $13 \mathrm{~N}-5.6 \mathrm{P}-10.9 \mathrm{~K}(13-13-13)$ at rates of 4.9 $\mathrm{g} \mathrm{N} / \mathrm{m}^{2}$ and $9.8 \mathrm{~g} \mathrm{~N} / \mathrm{m}^{2}$, Polyon ${ }^{\circledR} 13 \mathrm{~N}-5.6 \mathrm{P}-10.9 \mathrm{~K}(13-13-13)$ at rates of $4.9 \mathrm{~g} \mathrm{~N} / \mathrm{m}^{2}$ and $9.8 \mathrm{~g} \mathrm{~N} /$ $\mathrm{m}^{2}$, and CPL at rates of $4.9 \mathrm{~g} \mathrm{~N} / \mathrm{m}^{2}, 9.8 \mathrm{~g} \mathrm{~N} / \mathrm{m}^{2}, 19.6 \mathrm{~g} \mathrm{~N} / \mathrm{m}^{2}, 29.4 \mathrm{~g} \mathrm{~N} / \mathrm{m}^{2}, 39.2 \mathrm{~g} \mathrm{~N} / \mathrm{m}^{2}$, and $49 \mathrm{~g}$ $\mathrm{N} / \mathrm{m}^{2}$. Use of CPL incorporated into landscape planting beds as a fertilizer source resulted in plants equal to or larger than plants grown with conventional inorganic fertilizers. Nitrate $\left(\mathrm{NO}_{3}\right)$ and ammonia $\left(\mathrm{NH}_{4}\right)$ levels in leachates from plots amended with $\mathrm{CPL}$ were comparable with plots amended with commercial inorganic fertilizers and nitrogen $(\mathrm{N})$ levels were in most cases less in plots fertilized with CPL when compared with inorganic fertilizers when the same $\mathbf{N}$ rate was applied. Composted poultry litter may not be able to fully replace inorganic fertilizers, but it can reduce inorganic fertilizer requirements and provide an environmentally sound alternative to poultry waste disposal as well as provide beneficial aspects for plant growth in annual bedding plants.
\end{abstract}

Historically, poultry wastes have been used as a soil amendment and source of fertilizer in agronomic row cropping systems. Poultry litter (PL) is often preferred over other animal manures as a result of its high nutrient content in comparison with other animal manures. Typical PL will have the following nutrient ranges: $\mathrm{N}$ from $2.1 \%$ to $6 \%$, phosphorus $(\mathrm{P})$ from $1.4 \%$ to $9 \%$, potassium $(\mathrm{K})$ from $0.8 \%$ to $6.2 \%$, calcium $(\mathrm{Ca})$ from $0.8 \%$ to $6.1 \%$, magnesium $(\mathrm{Mg})$ from $0.2 \%$ to $2.1 \%$, and sulfur from $0.1 \%$ to $0.8 \%$ on a dry weight basis (Mitchell et al., 1995).

Land application was the predominant disposal method for PL in the past. However, as a result of increased environmental awareness, PL is now under strict new state and federal regulations concerning disposal methods with respect to non-point source pollution and other environmental concerns (Ribaudo, 2003). Because leached nitrate-N from landscape fertil-

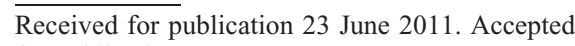
for publication 10 Aug. 2011.

${ }^{1}$ Graduate Research Assistant.

${ }^{2}$ Professor of Horticulture.

${ }^{3}$ Soil Scientist and Research Leader.

${ }^{4}$ To whom reprint requests should be addressed; e-mail sibleje@auburn.edu.
}

is the primary raw material used to produce ammonia, the rise in U.S. gas prices has led to a $35 \%$ decline in U.S. ammonia production capacity and a $44 \%$ decrease in output between years 2000 and 2006 (Wen-yuan, 2007). National average fertilizer prices increased $113 \%$ between 2000 and 2007 as a result of increases in N costs (Wen-yuan, 2007). During this 7-year period, the price of ammonia, the main source of $\mathrm{N}$ in fertilizer production, increased $130 \%$ and the price of urea, the primary solid $\mathrm{N}$ fertilizer used in the United States, rose $127 \%$ (Wen-yuan, 2007). As a result of price increases in inorganic fertilizer sources, the nutrients in PL are more valuable than ever.

There is potential for the use of organic manure-type products in the landscape and homeowner sector. Increased awareness of environmental impacts from the manufacture and use of inorganic fertilizer sources has caused many homeowners to seek more environmentally friendly products. Organically based products are becoming more popular, especially with urban consumers (Altland et al., 2002; Dimitri and Greene, 2002). Recent trends such as "Going Green" have made the use of organic products more desirable and marketable.

Poultry litter application has been shown to be beneficial as a fertilizer in row cropping systems (Burmester et al., 1991; Porch et al., 1990) and also has been shown to improve growth in roses, woody ornamentals, annuals, and bermuda grass production (Feagley et al., 2005; Reeder et al., 1992; Warren and Safley, 1990). In two experiments by Altland et al. (2003), growth of 'Magestic Giants White' pansy (Viola $\times$ wittrockiana) and Telstar Purple dianthus (Dianthus chinensis L.) was compared when fertilized with granular water-soluble fertilizers, controlled-release fertilizers, and an organically based fertilizer composed of recycled newspaper amended with PL at similar rates. Results indicated that plants grown using the organically based fertilizer grew as well or better than those using inorganic fertilizer treatments; however, the organic fertilizer caused elevated levels of total $\mathrm{N}$ in soil water in Expt. 2. However, composting PL before application could reduce $\mathrm{N}$ leaching. Use of CPL as fertilizer in urban landscapes could provide an environmentally sound means of disposal for poultry producers as well as a beneficial use for CPL when used as a replacement for inorganic fertilizers.

Research is needed to understand how the type of fertilizer used will affect plant growth and nutrient leaching. The objective of this study was to evaluate CPL as a fertilizer source for bedding plants at various rates in comparison with commercially available inorganic fertilizers for two commonly used landscape annual bedding species, 'Quartz Scarlet' verbena and 'Celebrity Red' petunia, in regard to plant growth and nutrient leaching.

\section{Materials and Methods} sionals, farmers, and turfgrass managers could begin using PL as an alternative or supplement to costly inorganic fertilizers. Inorganic fertilizer cost is increasing, whereas U.S. fertilizer production is decreasing. Because natural gas

\section{Expt. 1}

A fallow site (clay-loam soil) was chosen that had not received any fertilizer in $\approx 5$ 
years. The area was amended with $3.8 \mathrm{~cm}$ of milled pine bark and tilled to a $15.2-\mathrm{cm}$ depth. Raised beds were developed using a Kenco bed maker (Kenco Corp., Ligonier Valley, PA). Beds were divided into plots each being $3.05 \mathrm{~m} \times 0.46 \mathrm{~m}$ with $0.3 \mathrm{~m}$ between plots. Before planting, 10 treatments were applied: Peafowl ${ }^{\circledR}$ brand (Piedmont Fertilizer Co., Opelika, AL) garden-grade fertilizer $13 \mathrm{~N}-5.6 \mathrm{P}-10.9 \mathrm{~K}(13-13-13)$ at rates of $4.9 \mathrm{~g} \mathrm{~N} / \mathrm{m}^{2}$ (Pea1) and $9.8 \mathrm{~g} \mathrm{~N} / \mathrm{m}^{2}$ (Pea2), Polyon ${ }^{\circledR}$ (Agrium Advanced Technologies, Sylacauga, AL) (8- to 9-month controlledrelease fertilizer) at rates of $4.9 \mathrm{~g} \mathrm{~N} / \mathrm{m}^{2}$ (Poly1) and $9.8 \mathrm{~g} \mathrm{~N} / \mathrm{m}^{2}$ (Poly2), and CPL at rates of $4.9 \mathrm{~g} \mathrm{~N} / \mathrm{m}^{2}$ (CPL1), $9.8 \mathrm{~g} \mathrm{~N} / \mathrm{m}^{2}$ (CPL2), $19.6 \mathrm{~g}$ $\mathrm{N} / \mathrm{m}^{2}$ (CPL4), $29.4 \mathrm{~g} \mathrm{~N} / \mathrm{m}^{2}$ (CPL6), $39.2 \mathrm{~g} \mathrm{~N} / \mathrm{m}^{2}$ (CPL8), and $49 \mathrm{~g} \mathrm{~N} / \mathrm{m}^{2}$ (CPL10). A control receiving no fertilizer was also maintained for comparison. The $\mathrm{N}$ rate of $4.9 \mathrm{~g} \mathrm{~N} / \mathrm{m}^{2}$ used in this study was based on the Alabama Cooperative Extension Service's recommendation for herbaceous ornamentals in the landscape of applying $4.9 \mathrm{~g} \mathrm{~N} / \mathrm{m}^{2}$ of a complete fertilizer at planting (Williams and Mitchell, 1997). Additional rates of inorganic fertilizer $(9.8 \mathrm{~g}$ $\left.\mathrm{N} / \mathrm{m}^{2}\right)$ and CPL $\left(9.8 \mathrm{~g} \mathrm{~N} / \mathrm{m}^{2}, 19.6 \mathrm{~g} \mathrm{~N} / \mathrm{m}^{2}\right.$, $29.4 \mathrm{~g} \mathrm{~N} / \mathrm{m}^{2}, 39.2 \mathrm{~g} \mathrm{~N} / \mathrm{m}^{2}$, and $49.0 \mathrm{~g} \mathrm{~N} / \mathrm{m}^{2}$ ) were included to test the hypothesis that increasing $\mathrm{N}$ rate would increase crop growth and $\mathrm{N}$ leaching. Treatments were top-dressed on the prepared landscape beds and CPL was lightly raked in by hand before planting. Poultry litter used in this experiment was obtained from Greenville, AL, and was composted in an in-vessel rotating drum digester (BW Organics, Sulfur Springs, TX) for 2 weeks and stored under a tarp for $30 \mathrm{~d}$ until installation of the study. The CPL was analyzed by Brookside Laboratories Inc. (New Knoxville, $\mathrm{OH})$. Total $\mathrm{N}$ was determined using combustion analysis on the Elementar Vario max $\mathrm{CN}$ instrument (Elementar Analysensysteme, Hanau, Germany). Phosphorus and K content was determined using nitric acid digestion in a CEM Mars Express microwave (CEM Corporation, Matthews, NC) and analysis completed using a Thermo Scientific iCAP 6500 ICP spectrometer (ThermoFisher Scientific Inc., Waltham, MA). Analysis of CPL showed $3.8 \mathrm{~N}-1.0 \mathrm{P}-2.3 \mathrm{~K}\left[3.8 \%\right.$ total $\mathrm{N}\left(0.5 \% \mathrm{NH}_{4}\right)$, $2.2 \% \mathrm{P}$, and $2.8 \% \mathrm{~K}]$ on a dry weight basis. On 17 Aug. 2007, fertilizer treatments were applied to the raised beds and Petunia spp. 'Celebrity Red' and Verbena hybrida 'Quartz Scarlet' were selected for uniformity [both species $\approx 9 \mathrm{~cm}$ (height) $\times 5 \mathrm{~cm}$ (width)] and transplanted from 36 cell-pack liners into the prepared landscape beds. In each plot, 12 plants of one species were planted $0.3 \mathrm{~m}$ on center and arranged by species in a randomized complete block design with four replications per fertilizer treatment per species. Immediately after planting, all beds were mulched with pinestraw (2.5-cm depth). Plants received irrigation using overhead impact sprinklers as needed, and plots were hand-weeded as needed. Soil water leachates were collected using suction cup lysimeters (Soil Moisture Equipment Co., Santa Barbara, CA) $0.6 \mathrm{~m}$ long and $5.1 \mathrm{~cm}$ in diameter with a ceramic cap $7.6 \mathrm{~cm}$ long and $5.1 \mathrm{~cm}$ wide. Four lysimeters per fertilizer treatment were installed according to the manufacturer's recommendations (Soil Moisture Equipment Co., 2007) at a depth of $40.6 \mathrm{~cm}$ directly under plant roots. The hole for the lysimeter was formed using a soil core remover. A mud slurry using soil from the hole was poured back into the hole before insertion of the lysimeter to ensure soil contact with the ceramic cap according to the methods described by Altland et al. (2003). A 2005G2 vacuum test hand pump and 1900K3 Extraction kit (Soil Moisture Equipment Co.) were used to create a suction of 60 centibars ( $8.82 \mathrm{psi})$ within the lysimeter $24 \mathrm{~h}$ before sample collection.

Data were collected on four plants in the center of each replicate for each species to avoid any edge effects from the treatment plots. Data collected included growth indices $[($ height + width $1+$ width 2$) / 3(\mathrm{~cm})]$ at 4 and 8 weeks after planting (WAP) as well as leaf chlorophyll content (LCC) on four leaves per plant using a SPAD-502 Chlorophyll Meter (Spectrum Technologies, Inc., Plainfield, IL) at 4, 8, and 12 WAP. Plant shoot dry weights were recorded at the conclusion of the study (12 WAP) after drying in a forced-air oven at $70^{\circ} \mathrm{C}$ for $72 \mathrm{~h}$. Leachates were collected using lysimeters at 2, 4, 8, 12, and 16 WAP. Soil water collected from lysimeters was analyzed using a colorimetric procedure (Sims et al., 1995) to determine $\mathrm{N}$ as $\mathrm{NO}_{3}$ and $\mathrm{NH}_{4}$. Water was also analyzed for $\mathrm{pH}$ and electrical conductivity $\left(\mathrm{dS} \cdot \mathrm{m}^{-1}\right)$. Foliar samples (most recently matured leaves) (Mills and Jones, 1996) were collected and analyzed for nutrient content $(\mathrm{N}, \mathrm{P}$, $\mathrm{K}, \mathrm{Ca}, \mathrm{Mg}$, boron, iron, manganese, copper, zinc, aluminum, and sodium) at 12 WAP. Foliar $\mathrm{N}$ was determined by combustion analysis using a $1500 \mathrm{~N}$ analyzer (Carlo Erba, Milan, Italy). Remaining nutrients were determined by microwave digestion with inductively coupled plasmaemission spectrometry (Thermo Jarrel Ash, Offenbach, Germany). Data were subjected to analysis of variance with means separation by Duncan's multiple range test $(P \leq 0.05)$ using the proc GLM feature in a statistical software package (SAS ${ }^{\circledast}$ Institute Version 9.1, Cary, NC).
Expt. 2

Expt. 2 was similar to Expt. 1 with the following exceptions. On 19 May 2008, fertilizer treatments were applied to the raised landscape beds and plants were planted like in Expt. 1. Composted poultry litter analysis by Brookside Laboratories Inc. (New Knoxville, $\mathrm{OH}$ ) showed $3.6 \mathrm{~N}-1.1 \mathrm{P}-4.1 \mathrm{~K}[3.6 \%$ total N $(0.4 \%$ as $\left.\mathrm{NH}_{4}\right), 2.6 \% \mathrm{P}$, and $4.9 \% \mathrm{~K}$ ] on a dry weight basis. Leachates were collected using lysimters at $1,2,4,8$, and 12 WAP.

\section{Results}

\section{Expt. 1}

Growth indices and dry weights. At 4 WAP, petunia growth was similar among Peafowl, Polyon, and CPL treatments when the same rate of $\mathrm{N}$ was applied (Table 1). By 8 WAP, petunias fertilized using CPL1 were as large or larger than those fertilized with either inorganic fertilizer at the same $\mathrm{N}$ rate. In addition, Pea1 and Poly1 had similar growth indices as the non-fertilized control. Although plants fertilized with CPL1 had larger growth indices (GI) than those in Pea1 or Poly1 treatments, Pea2, Poly2, and CPL2 had similar GI at 8 WAP. Plants fertilized with CPL8 had higher GI than any other treatment with the exception of plants in Pea2, CPL6 and CPL10, which were similar. Petunia dry weight (DW) revealed that petunias grown in CPL plots had similar DW as petunias grown with Peafowl or Polyon fertilizers when the same $\mathrm{N}$ rate of fertilizer was applied. Petunias fertilized with Peafowl (both rates) and Poly 2 had similar DW to CPL treatments regardless of rate, whereas plants grown using Polyl had less DW than Pea2, CPL6, CPL8, and CPL10 treatments.

Verbena GI revealed that Polyon fertilizers (both rates) and Pea1 had similar GI to CPL treatments at $4 \mathrm{WAP}$ when the same $\mathrm{N}$ rate was applied; however, Pea2 was larger than CPL2 (Table 1). Verbena DW showed that similar to petunia, when the same $\mathrm{N}$ rate of fertilizer was used, plants grown with CPL were similar to Peafowl or Polyon treatments. Furthermore, all fertilizer treatments had similar DW with the exception of

Table 1. Effect of fertilizers on growth indices ${ }^{z}$ and shoot dry weights ${ }^{y}$ of 'Celebrity Red' petunia and 'Quartz Scarlet' verbena, Expt. 1.

\begin{tabular}{|c|c|c|c|c|c|c|c|}
\hline \multirow[b]{2}{*}{ Treatment } & \multirow{2}{*}{$\begin{array}{c}\text { Rate } \\
\left(\mathrm{g} \mathrm{N} / \mathrm{m}^{2}\right)\end{array}$} & \multicolumn{3}{|c|}{ 'Celebrity Red' Petunia } & \multicolumn{3}{|c|}{ 'Quartz Scarlet' Verbena } \\
\hline & & $4 \mathrm{WAP}^{\mathrm{x}}$ & 8 WAP & Dry wt & 4 WAP & 8 WAP & Dry wt \\
\hline 13-13-13 Peafowl® & 4.9 & $13.5 \mathrm{bc}^{\mathrm{w}}$ & $18.6 \mathrm{~cd}$ & $123.8 \mathrm{abc}$ & $17.1 \mathrm{ab}$ & $21.0 \mathrm{bc}$ & $93.6 \mathrm{bc}$ \\
\hline 13-13-13 Peafowl@ & 9.8 & $15.3 \mathrm{ab}$ & $22.2 \mathrm{ab}$ & $150.4 \mathrm{a}$ & $18.7 \mathrm{a}$ & $22.7 \mathrm{ab}$ & $113.2 \mathrm{ab}$ \\
\hline 13-13-13 Polyon® & 4.9 & $12.5 \mathrm{~cd}$ & $16.8 \mathrm{~d}$ & $92.1 \mathrm{bc}$ & $16.1 \mathrm{~b}$ & $22.3 \mathrm{abc}$ & $111.0 \mathrm{ab}$ \\
\hline 13-13-13 Polyon $\AA$ & 9.8 & $13.6 \mathrm{abc}$ & $20.8 \mathrm{bc}$ & $127.3 \mathrm{ab}$ & $16.6 \mathrm{ab}$ & $23.1 \mathrm{ab}$ & $109.7 \mathrm{ab}$ \\
\hline $\mathrm{CPL}^{\mathrm{v}}$ & 4.9 & $13.4 \mathrm{bc}$ & $20.6 \mathrm{bc}$ & $109.2 \mathrm{bc}$ & $15.6 \mathrm{~b}$ & $22.6 \mathrm{ab}$ & $107.7 \mathrm{ab}$ \\
\hline CPL & 9.8 & $14.2 \mathrm{abc}$ & $21.6 \mathrm{bc}$ & $121.9 \mathrm{abc}$ & $15.4 \mathrm{~b}$ & $22.8 \mathrm{ab}$ & $94.0 \mathrm{bc}$ \\
\hline CPL & 19.6 & $15.5 \mathrm{a}$ & $20.8 \mathrm{bc}$ & $126.9 \mathrm{ab}$ & $16.1 \mathrm{~b}$ & $23.2 \mathrm{ab}$ & $117.1 \mathrm{ab}$ \\
\hline CPL & 29.4 & $14.4 \mathrm{ab}$ & $23.6 \mathrm{ab}$ & $147.6 \mathrm{a}$ & $17.1 \mathrm{ab}$ & $22.9 \mathrm{ab}$ & $112.5 \mathrm{ab}$ \\
\hline CPL & 39.2 & $15.1 \mathrm{ab}$ & $25.0 \mathrm{a}$ & $150.2 \mathrm{a}$ & $17.7 \mathrm{ab}$ & $23.7 \mathrm{ab}$ & $122.5 \mathrm{ab}$ \\
\hline CPL & 49 & $13.8 \mathrm{abc}$ & $22.9 \mathrm{ab}$ & $156.5 \mathrm{a}$ & $16.3 \mathrm{~b}$ & $24.1 \mathrm{a}$ & $126.1 \mathrm{a}$ \\
\hline Non-fertilized control & $* * *$ & $11.3 \mathrm{~d}$ & $16.0 \mathrm{~d}$ & $90.1 \mathrm{c}$ & $15.7 \mathrm{~b}$ & $19.8 \mathrm{c}$ & $78.6 \mathrm{c}$ \\
\hline
\end{tabular}

${ }^{\mathrm{z}}$ Growth indices $=[($ height + width $1+$ width2 $) / 3]$.

y Dry weights measured in grams taken from average dry weight of five plants per plot from each replication.

${ }^{\mathrm{x}} \mathrm{WAP}=$ Weeks after planting.

"Means separated within columns using Duncan's mutliple range test $(\alpha=0.05)$.

${ }^{\mathrm{v}} \mathrm{CPL}=$ Composted poultry litter. 
CPL10, which had higher DW than Pea1 or CPL2.

Leaf chlorophyll content. At 4 WAP there were no differences in LCC of petunia in any treatment (Table 2). At 8 and $12 \mathrm{WAP}, \mathrm{LCC}$ was similar among Peafowl, Polyon, and CPL treatments when the same $\mathrm{N}$ rate was applied. At 12 WAT, all fertilizer treatments had similar LCC with the exception of petunias grown with CPL10, which had higher LCC than petunias in Pea1, Poly2, CPL1, CPL2, and CPL4.

No differences were observed in LCC of verbena at 4,8 , or 12 WAP in plots receiving a fertilization treatment. nia tissue nutrient content was similar among all treatments for $\mathrm{N}$ with Peal having slightly less foliar $\mathrm{N}$ and Poly2 having slightly more (data not shown). Phosphorus levels tended to be greater in the highest CPL treatments (CPL6, CPL8, and CPL10); however, P levels were similar among CPL and Peafowl and Polyon fertilizers when the same $\mathrm{N}$ rate was applied. No differences were observed in $\mathrm{K}$ among any treatment. All other essential nutrients were equal to or above sufficiency ranges with the exception of boron, which was slightly less than the sufficiency range (Mills and Jones, 1996).

There were no differences in foliar $\mathrm{N}$ content of verbena (data not shown). Again,
Tissue nutrient content. In general, petu-

$\mathrm{P}$ tended to be highest in treatments fertilized with CPL8 and CPL10. Potassium content was highest in verbena fertilized with Pea1; however, foliar K content was similar to both rates of Polyon and CPL2, CPL6, CPL8, and CPL10. All other essential nutrients were within or above sufficiency levels (Mills and Jones, 1996).

Nitrogen levels in soil water as $\mathrm{NO}_{3}-\mathrm{N}$ and $\mathrm{NH}_{4}-\mathrm{N}$. At $2 \mathrm{WAP}, \mathrm{NO}_{3}$ and $\mathrm{NH}_{4}$ were high for all treatments (Table 3 ). No treatment was less than six times the Environmental Protection Agency (EPA)-recommended level for $\mathrm{NO}_{3}$ in drinking water supplies $\left(10 \mathrm{mg} \cdot \mathrm{L}^{-1}\right)$ (EPA, 1972, 2009). At 4 WAP, $\mathrm{NO}_{3}$ levels remained high; $\mathrm{NH}_{4}$ levels began to drop and no differences were observed among any treatment with the exception of Pea2, which was significantly higher than all other treatments, a trend that continued at 8 WAP. However, at 8 weeks, $\mathrm{NO}_{3}$ dropped significantly from previous levels and all treatments were within acceptable levels for drinking water with the exception of the Peafowl fertilizer (both rates), which remained high. By $12 \mathrm{WAP}, \mathrm{NO}_{3}$ and $\mathrm{NH}_{4}$ levels were below $10 \mathrm{mg} / \mathrm{L}$ and remained within these recommended levels for the duration of the study. Although all treatments were within recommended levels, Polyon at the $9.8 \mathrm{~g} \mathrm{~N} / \mathrm{m}^{2}$ rate had higher $\mathrm{NO}_{3}$ levels at 12 and 16 WAP than other treatments, possibly

Table 2. Effect of fertilizers on leaf chlorophyll content ${ }^{\mathrm{z}}$ of 'Celebrity Red' petunia and 'Quartz Scarlet' verbena, Expt. 1.

\begin{tabular}{|c|c|c|c|c|c|c|c|}
\hline \multirow[b]{2}{*}{ Treatment } & \multirow{2}{*}{$\begin{array}{c}\text { Rate } \\
\left(\mathrm{g} \mathrm{N} / \mathrm{m}^{2}\right)\end{array}$} & \multicolumn{3}{|c|}{ 'Celebrity Red' Petunia } & \multicolumn{3}{|c|}{ 'Quartz Scarlet' Verbena } \\
\hline & & $4 \mathrm{WAP}^{\mathrm{y}}$ & 8 WAP & $12 \mathrm{WAP}$ & 4 WAP & $8 \mathrm{WAP}$ & $12 \mathrm{WAP}$ \\
\hline 13-13-13 Peafowl ${ }^{1}$ & 4.9 & $48.8 \mathrm{a}^{\mathrm{x}}$ & $49.0 \mathrm{abc}$ & $38.1 \mathrm{c}$ & $45.6 \mathrm{a}$ & $44.7 \mathrm{a}$ & $43.0 \mathrm{a}$ \\
\hline 13-13-13 Peafowl® & 9.8 & $48.4 \mathrm{a}$ & $48.9 \mathrm{abc}$ & $43.5 \mathrm{ab}$ & $49.0 \mathrm{a}$ & $44.4 \mathrm{a}$ & $42.9 \mathrm{a}$ \\
\hline-13 Polyon ${ }^{\circledR}$ & 4.9 & $46.9 \mathrm{a}$ & $48.2 \mathrm{abc}$ & $42.7 \mathrm{ab}$ & $46.6 \mathrm{a}$ & & $45.2 \mathrm{a}$ \\
\hline $13-13-1$ & 9.8 & $48.9 \mathrm{a}$ & $49.5 \mathrm{ab}$ & $42.0 \mathrm{bc}$ & $47.8 \mathrm{a}$ & 46.4 & $42.5 \mathrm{a}$ \\
\hline $\mathrm{CPL}^{\mathrm{w}}$ & 4.9 & $46.5 \mathrm{a}$ & $44.4 \mathrm{c}$ & $40.4 \mathrm{bc}$ & $45.2 \mathrm{a}$ & $43.0 \mathrm{ab}$ & $40.4 \mathrm{ab}$ \\
\hline CPL & 9.8 & $49.2 \mathrm{a}$ & $46.9 \mathrm{bc}$ & $41.8 \mathrm{bc}$ & $45.1 \mathrm{a}$ & 41.5 & $44.3 \mathrm{a}$ \\
\hline CPL & 19.6 & $46.6 \mathrm{a}$ & $46.3 \mathrm{bc}$ & $40.5 \mathrm{bc}$ & $44.2 \mathrm{a}$ & $43.2 \mathrm{ab}$ & $42.4 \mathrm{a}$ \\
\hline CPL & 29.4 & $48.0 \mathrm{a}$ & $46.2 \mathrm{bc}$ & $44.1 \mathrm{ab}$ & $45.0 \mathrm{a}$ & $45.3 \mathrm{a}$ & $43.3 \mathrm{a}$ \\
\hline CPL & 39.2 & $47.9 \mathrm{a}$ & $47.5 \mathrm{abc}$ & $44.2 \mathrm{ab}$ & $47.5 \mathrm{a}$ & $45.8 \mathrm{a}$ & $42.0 \mathrm{a}$ \\
\hline CPL & 49 & $49.8 \mathrm{a}$ & $51.9 \mathrm{a}$ & $46.7 \mathrm{a}$ & $46.4 \mathrm{a}$ & $47.2 \mathrm{a}$ & $44.7 \mathrm{a}$ \\
\hline Non-fertilized control & $* * *$ & $47.2 \mathrm{a}$ & $46.0 \mathrm{bc}$ & $41.9 \mathrm{bc}$ & $45.0 \mathrm{a}$ & $38.3 \mathrm{~b}$ & $36.2 \mathrm{~b}$ \\
\hline
\end{tabular}

${ }^{2}$ Leaf chlorophyll content quantified using a SPAD-502 Chlorophyll Meter (Minolta Camera Co., Ramsey, $\mathrm{NJ})$ (average of five leaves per plant).

${ }^{\mathrm{y}} \mathrm{WAP}=$ Weeks after planting.

${ }^{x}$ Means within column followed by the same letter are not significantly different based on Duncan's multiple range test $(\alpha=0.05)$.

${ }^{\mathrm{w}} \mathrm{CPL}=$ Composted poultry litter. because it is a controlled-release fertilizer (CRF). This indicates that although CRF may have similar $\mathrm{N}$ levels to other fertilizers (organic or inorganic) in soil water after initial application, the release curve for $\mathrm{N}$ leaching may shift to later.

Water $\mathrm{pH}$ and electrical conductivity. There were no differences in soil solution $\mathrm{pH}$ among any treatment throughout the study (data not shown). Electrical conductivity (EC) level of Pea2 was significantly higher than all other treatments at 2 WAP (data not shown). At 4 WAP, Pea2, and CPL4, CPL6, CPL8, and CPL10 had the highest EC levels. By 8 WAP, Pea2 had the highest EC levels among any treatment. At 12 WAP, CPL8 had higher EC levels than Poly1, CPL1, CPL2, and CPL6. At the conclusion of the study (16 WAP), there were no differences in EC levels among fertilized plots.

\section{Expt. 2}

Growth indices and dry weights. Petunia GI at 4 WAP indicated that Peafowl fertilizer at both rates along with CPL4, CPL6, CPL8, and CPL10 grew the largest plants, a trend that continued at 8 WAP (Table 4). Like in Expt. 1, petunia DW indicated that plants fertilized with CPL were similar to plants fertilized with inorganic fertilizers at the same rate.

At 4 WAP, verbena fertilized with Pea2 along with CPL4, CPL6, CPL8, and CPL10 had the largest GI. At 8 WAP, few differences were seen in verbena GI, and all plants fertilized with CPL had growth similar to plants fertilized with inorganic fertilizer regardless of the rate.

Verbena DW indicated that plants grew similarly when fertilized with Peafowl, Polyon, or CPL when the same $\mathrm{N}$ rate was applied. However, plants fertilized with the highest rates of CPL $\left(29.4,39.2\right.$, and $\left.49.0 \mathrm{~g} \mathrm{~N} / \mathrm{m}^{2}\right)$ were similar to or smaller than inorganic (Peafowl or Polyon) fertilizer treatments.

Leaf chlorophyll content. Petunia LCC was generally similar throughout the study; however, small differences did occur (Table 5). At 4 WAP, plants in Pea 2 had higher LCC than plants in Poly1, Poly2, CPL1, CPL2, CPL4, and CPL8. Leaf chlorophyll content was similar among most treatments at $8 \mathrm{WAP}$; however, by 12 WAP, CPL1 had a higher

Table 3. Effect of fertilizers on nitrogen (presented as $\mathrm{mg} / \mathrm{L}$ of $\mathrm{NO}_{3}$ and $\mathrm{NH}_{4}$ ) in soil water, Expt. 1.

\begin{tabular}{|c|c|c|c|c|c|c|c|c|c|c|c|}
\hline \multirow[b]{2}{*}{ Treatment } & \multirow[b]{2}{*}{ Rate $\left(\mathrm{g} \mathrm{N} / \mathrm{m}^{2}\right)$} & \multicolumn{2}{|c|}{$2 \mathrm{WAP}^{\mathrm{z}}$} & \multicolumn{2}{|c|}{4 WAP } & \multicolumn{2}{|c|}{$8 \mathrm{WAP}$} & \multicolumn{2}{|c|}{12 WAP } & \multicolumn{2}{|c|}{16 WAP } \\
\hline & & $\mathrm{NO}_{3}$ & $\mathrm{NH}_{4}$ & $\mathrm{NO}_{3}$ & $\mathrm{NH}_{4}$ & $\mathrm{NO}_{3}$ & $\mathrm{NH}_{4}$ & $\mathrm{NO}_{3}$ & $\mathrm{NH}_{4}$ & $\mathrm{NO}_{3}$ & $\mathrm{NH}_{4}$ \\
\hline 13-13-13 Peafowl® & 4.9 & $121.0 \mathrm{ab}$ & $27.1 \mathrm{a}^{\mathrm{y}}$ & $111.3 \mathrm{bcd}$ & $3.9 \mathrm{~b}$ & $22.9 \mathrm{~b}$ & $0.87 \mathrm{~b}$ & $0.87 \mathrm{~b}$ & $0.10 \mathrm{ab}$ & $0.27 \mathrm{~b}$ & $0.17 \mathrm{a}$ \\
\hline 13-13-13 Peafowl $®$ & 9.8 & $76.4 \mathrm{bcd}$ & $5.3 \mathrm{~b}$ & $141.6 \mathrm{abc}$ & $44.7 \mathrm{a}$ & $87.1 \mathrm{a}$ & $11.3 \mathrm{a}$ & $1.73 \mathrm{~b}$ & $0.17 \mathrm{a}$ & $0.50 \mathrm{~b}$ & $0.10 \mathrm{a}$ \\
\hline 13-13-13 Polyon ${ }^{\circledR}$ & 4.9 & $62.5 \mathrm{~d}$ & $4.0 \mathrm{~b}$ & $74.9 \mathrm{~cd}$ & $0.4 \mathrm{~b}$ & $0.93 \mathrm{~b}$ & $0.07 \mathrm{~b}$ & $0.20 \mathrm{~b}$ & $0.03 \mathrm{~b}$ & $0.23 \mathrm{~b}$ & $0.07 \mathrm{a}$ \\
\hline 13-13-13 Polyon $\AA$ & 9.8 & $90.5 \mathrm{abcd}$ & $12.9 \mathrm{ab}$ & $95.3 \mathrm{bcd}$ & $0.9 \mathrm{~b}$ & $8.9 \mathrm{~b}$ & $0.10 \mathrm{~b}$ & $3.27 \mathrm{a}$ & $0.17 \mathrm{a}$ & $2.4 \mathrm{a}$ & $0.10 \mathrm{a}$ \\
\hline $\mathrm{CPL}^{\mathrm{x}}$ & 4.9 & $103.2 \mathrm{abcd}$ & $3.0 \mathrm{~b}$ & $54.9 \mathrm{~d}$ & $0.4 \mathrm{~b}$ & $0.80 \mathrm{~b}$ & $0.17 \mathrm{~b}$ & $0.83 \mathrm{~b}$ & $0.03 \mathrm{~b}$ & $0.20 \mathrm{~b}$ & $0.17 \mathrm{a}$ \\
\hline CPL & 9.8 & $73.8 \mathrm{~cd}$ & $9.3 \mathrm{ab}$ & $102.6 \mathrm{bcd}$ & $0.7 \mathrm{~b}$ & $6.0 \mathrm{~b}$ & $0.13 \mathrm{~b}$ & $0.30 \mathrm{~b}$ & $0.0 \mathrm{~b}$ & $0.13 \mathrm{~b}$ & $0.10 \mathrm{a}$ \\
\hline CPL & 19.6 & $118.2 \mathrm{abc}$ & $7.4 \mathrm{ab}$ & $194.4 \mathrm{a}$ & $2.8 \mathrm{~b}$ & $2.9 \mathrm{~b}$ & $0.17 \mathrm{~b}$ & $0.63 \mathrm{~b}$ & $0.03 \mathrm{~b}$ & $1.0 \mathrm{ab}$ & $0.27 \mathrm{a}$ \\
\hline $\mathrm{CPL}$ & 29.4 & $79.7 \mathrm{bcd}$ & $7.0 \mathrm{ab}$ & $104.8 \mathrm{bcd}$ & $1.1 \mathrm{~b}$ & $3.7 \mathrm{~b}$ & $0.10 \mathrm{~b}$ & $0.97 \mathrm{~b}$ & $0.07 \mathrm{ab}$ & $0.87 \mathrm{ab}$ & $0.13 \mathrm{a}$ \\
\hline CPL & 39.2 & $128.6 \mathrm{a}$ & $5.5 \mathrm{~b}$ & $129.0 \mathrm{abcd}$ & $1.6 \mathrm{~b}$ & $1.3 \mathrm{~b}$ & $0.23 \mathrm{~b}$ & $0.67 \mathrm{~b}$ & $0.06 \mathrm{ab}$ & $0.37 \mathrm{~b}$ & $0.13 \mathrm{a}$ \\
\hline CPL & 49 & $114.1 \mathrm{abc}$ & $21.7 \mathrm{ab}$ & $165.6 \mathrm{ab}$ & $3.1 \mathrm{~b}$ & $8.8 \mathrm{~b}$ & $0.27 \mathrm{~b}$ & $0.17 \mathrm{~b}$ & $0.0 \mathrm{~b}$ & $0.53 \mathrm{~b}$ & $0.20 \mathrm{a}$ \\
\hline Non-fertilized control & $* * *$ & $68.8 \mathrm{~d}$ & $0.9 \mathrm{~b}$ & $77.0 \mathrm{~cd}$ & $0.2 \mathrm{~b}$ & $1.3 \mathrm{~b}$ & $0.17 \mathrm{~b}$ & $0.50 \mathrm{~b}$ & $0.0 \mathrm{~b}$ & $0.53 \mathrm{~b}$ & $0.10 \mathrm{a}$ \\
\hline
\end{tabular}

${ }^{\mathrm{z}} \mathrm{WAP}=$ Weeks after planting.

${ }^{y}$ Means within column followed by the same letter are not significantly different based on Duncan's multiple range test $(\alpha=0.05)$

${ }^{\mathrm{x}} \mathrm{CPL}=$ Composted poultry litter. 
LCC than all other treatments except for the highest rates of CPL ( 39.2 and $49.0 \mathrm{~g} \mathrm{~N} / \mathrm{m}^{2}$ ).

Similar to petunia, verbena LCC at 4 WAP showed little differences between treatments (Table 5). However, by 8 WAP, Poly2 had higher LCC than any other treatment with the exception of Poly1, Pea2, and CPL4, which were similar. By 12 WAP, verbena fertilized with Poly2 had higher LCC than all other treatments.

Table 4. Effect of fertilizers on growth indices ${ }^{z}$ and shoot dry weights ${ }^{y}$ of 'Celebrity Red' petunia and 'Quartz Scarlet' verbena, Expt. 2.

\begin{tabular}{|c|c|c|c|c|c|c|c|}
\hline \multirow[b]{2}{*}{ Treatment } & \multirow[b]{2}{*}{ Rate $\left(\mathrm{g} \mathrm{N} / \mathrm{m}^{2}\right)$} & \multicolumn{3}{|c|}{ 'Celebrity Red' Petunia } & \multicolumn{3}{|c|}{ 'Quartz Scarlet' Verbena } \\
\hline & & $4 \mathrm{WAP}^{\mathrm{x}}$ & 8 WAP & Dry wt & 4 WAP & 8 WAP & Dry wt \\
\hline 13-13-13 Peafowl® & 4.9 & $24.7 \mathrm{a}^{\mathrm{w}}$ & $38.3 \mathrm{a}$ & $231.7 \mathrm{ab}$ & $14.2 \mathrm{~cd}$ & $27.1 \mathrm{ab}$ & $189.4 \mathrm{abc}$ \\
\hline 13-13-13 Peafowl $®$ & 9.8 & $25.2 \mathrm{a}$ & $38.8 \mathrm{a}$ & $252.8 \mathrm{a}$ & $17.0 \mathrm{a}$ & $26.9 \mathrm{abc}$ & $187.5 \mathrm{abc}$ \\
\hline 13-13-13 Polyon® & 4.9 & $19.2 \mathrm{~cd}$ & $32.7 \mathrm{~b}$ & $221.0 \mathrm{ab}$ & $12.7 \mathrm{e}$ & $26.2 \mathrm{abc}$ & $191.7 \mathrm{abc}$ \\
\hline 13-13-13 Polyon $\AA$ & 9.8 & $17.8 \mathrm{~d}$ & $33.3 \mathrm{~b}$ & $233.5 \mathrm{ab}$ & $13.4 \mathrm{de}$ & $26.7 \mathrm{abc}$ & $233.8 \mathrm{a}$ \\
\hline $\mathrm{CPL}^{\mathrm{v}}$ & 4.9 & $19.5 \mathrm{c}$ & $32.2 \mathrm{~b}$ & $215.6 \mathrm{ab}$ & $14.2 \mathrm{~cd}$ & $28.1 \mathrm{a}$ & $224.9 \mathrm{ab}$ \\
\hline CPL & 9.8 & $21.4 \mathrm{~b}$ & $33.6 \mathrm{~b}$ & $226.7 \mathrm{ab}$ & $15.2 \mathrm{bc}$ & $27.3 \mathrm{ab}$ & $207.7 \mathrm{abc}$ \\
\hline CPL & 19.6 & $24.0 \mathrm{a}$ & $38.8 \mathrm{a}$ & $232.2 \mathrm{ab}$ & $16.2 \mathrm{ab}$ & $27.7 \mathrm{a}$ & $200.7 \mathrm{abc}$ \\
\hline CPL & 29.4 & $24.4 \mathrm{a}$ & $39.0 \mathrm{a}$ & $263.2 \mathrm{a}$ & $16.3 \mathrm{ab}$ & $26.7 \mathrm{abc}$ & $180.2 \mathrm{bc}$ \\
\hline CPL & 39.2 & $25.5 \mathrm{a}$ & $40.9 \mathrm{a}$ & $274.0 \mathrm{a}$ & $16.11 \mathrm{ab}$ & $25.3 \mathrm{bc}$ & $170.9 \mathrm{c}$ \\
\hline CPL & 49 & $25.0 \mathrm{a}$ & $41.0 \mathrm{a}$ & $262.5 \mathrm{a}$ & $17.0 \mathrm{a}$ & $26.3 \mathrm{abc}$ & $176.6 \mathrm{bc}$ \\
\hline Non-fertilized control & $* * *$ & $18.2 \mathrm{~cd}$ & $34.5 \mathrm{~b}$ & $177.9 \mathrm{~b}$ & $12.6 \mathrm{e}$ & $24.7 \mathrm{c}$ & $164.9 \mathrm{c}$ \\
\hline
\end{tabular}

${ }^{\mathrm{z}}$ Growth indices $=[($ height + width $1+$ width2 $) / 3]$.

${ }^{y}$ Dry weights measured in grams, taken from average dry weight of 5 plants per plot from each replication. ${ }^{\mathrm{x}} \mathrm{WAP}=$ Weeks after planting.

wMeans separated within columns using Duncan's mutliple range test $(\alpha=0.05)$.

${ }^{\mathrm{v}} \mathrm{CPL}=$ Composted poultry litter

Table 5. Effect of fertilizers on leaf chlorophyll content ${ }^{\mathrm{z}}$ of 'Celebrity Red' petunia and 'Quartz Scarlet' verbena, Expt. 2.

\begin{tabular}{|c|c|c|c|c|c|c|c|}
\hline \multirow[b]{2}{*}{ Treatment } & \multirow[b]{2}{*}{ Rate $\left(\mathrm{g} \mathrm{N} / \mathrm{m}^{2}\right)$} & \multicolumn{3}{|c|}{ 'Celebrity Red' Petunia } & \multicolumn{3}{|c|}{ 'Quartz Scarlet' Verbena } \\
\hline & & $\overline{4 \mathrm{WAP}^{\mathrm{y}}}$ & $8 \mathrm{WAP}$ & $12 \mathrm{WAP}$ & $4 \mathrm{WAP}$ & $8 \mathrm{WAP}$ & $12 \mathrm{WAP}$ \\
\hline$\overline{13-13-13 \text { Peafowl® }}$ & 4.9 & $46.0 \mathrm{ab}^{\mathrm{x}}$ & $36.3 \mathrm{bc}$ & $32.5 \mathrm{~b}$ & $53.1 \mathrm{abc}$ & $47.5 \mathrm{~b}$ & $36.2 \mathrm{bcd}$ \\
\hline 13-13-13 Peafowl® & 9.8 & $47.3 \mathrm{a}$ & $36.8 \mathrm{abc}$ & $32.7 \mathrm{~b}$ & $53.4 \mathrm{ab}$ & $48.7 \mathrm{ab}$ & $37.4 \mathrm{bcd}$ \\
\hline 13-13-13 Polyon® & 4.9 & $43.3 \mathrm{bcd}$ & $38.7 \mathrm{ab}$ & $35.8 \mathrm{~b}$ & $50.9 \mathrm{abc}$ & $48.7 \mathrm{ab}$ & $37.4 \mathrm{bcd}$ \\
\hline 13-13-13 Polyon $®$ & 9.8 & $43.7 \mathrm{bc}$ & $39.1 \mathrm{a}$ & $36.3 \mathrm{~b}$ & $50.4 \mathrm{bc}$ & $51.5 \mathrm{a}$ & $45.6 \mathrm{a}$ \\
\hline $\mathrm{CPL}^{\mathrm{w}}$ & 4.9 & $43.2 \mathrm{bcd}$ & $39.1 \mathrm{a}$ & $51.0 \mathrm{a}$ & $51.4 \mathrm{abc}$ & $48.4 \mathrm{~b}$ & $40.1 \mathrm{~b}$ \\
\hline $\mathrm{CPL}$ & 9.8 & $42.4 \mathrm{~cd}$ & $36.9 \mathrm{abc}$ & $35.5 \mathrm{~b}$ & $52.2 \mathrm{abc}$ & $46.1 \mathrm{~b}$ & $38.3 \mathrm{bcd}$ \\
\hline CPL & 19.6 & $42.6 \mathrm{~cd}$ & $37.0 \mathrm{abc}$ & $33.5 \mathrm{~b}$ & $49.8 \mathrm{c}$ & $49.2 \mathrm{ab}$ & $34.4 \mathrm{~cd}$ \\
\hline CPL & 29.4 & $45.4 \mathrm{abc}$ & $37.7 \mathrm{abc}$ & $36.4 \mathrm{~b}$ & $51.5 \mathrm{abc}$ & $48.3 \mathrm{~b}$ & $37.4 \mathrm{bcd}$ \\
\hline CPL & 39.2 & $44.1 \mathrm{bc}$ & $36.0 \mathrm{c}$ & $37.8 \mathrm{ab}$ & $54.3 \mathrm{a}$ & $48.2 \mathrm{~b}$ & $36.2 \mathrm{bcd}$ \\
\hline CPL & 49 & $44.8 \mathrm{abc}$ & $37.1 \mathrm{abc}$ & $38.1 \mathrm{ab}$ & $53.4 \mathrm{ab}$ & $47.5 \mathrm{~b}$ & $38.5 \mathrm{bc}$ \\
\hline Non-fertilized control & $* * *$ & $40.5 \mathrm{~d}$ & $35.4 \mathrm{c}$ & $31.0 \mathrm{~b}$ & $42.9 \mathrm{~d}$ & $47.6 \mathrm{~b}$ & $33.7 \mathrm{~d}$ \\
\hline
\end{tabular}

${ }^{\mathrm{z}}$ Leaf chlorophyll content quantified using a SPAD-502 Chlorophyll Meter (Minolta Camera Co., Ramsey, NJ) (average of five leaves per plant).

${ }^{\mathrm{y}} \mathrm{WAP}=$ Weeks after planting.

'Means within column followed by the same letter are not significantly different based on Duncan's multiple range test $(\alpha=0.05)$.

${ }^{\mathrm{w}} \mathrm{CPL}=$ Composted poultry litter.
Similar to petunia tissue nutrient content, little difference was observed in verbena $\mathrm{N}$ levels and all were above sufficiency range (1.43 to 1.90; Mills and Jones, 1996) (data not shown). CPL10 had higher leaf P content than all other treatments with the exception of CPL8, which was similar. Verbena K content was above sufficient levels in all treatments. All other required nutrients were present at or above the sufficiency range, with the exception of $\mathrm{Ca}$ and $\mathrm{B}$, which were slightly lower. Only minor differences occurred in micronutrient content with the exception of manganese, which generally decreased as the level of CPL increased.

Nitrogen levels in soil water as $\mathrm{NO}_{3}-\mathrm{N}$ and $\mathrm{NH}_{4}-\mathrm{N}$. At $1 \mathrm{WAP}$, there were minor differences in $\mathrm{NO}_{3}$ levels in water, and all treatments were equal to or less than the non-fertilized control (Table 6). Ammonium levels were also similar among all treatments, and the only significant difference occurred among Poly1, which had higher water $\mathrm{NH}_{4}$ levels than CPL2 plots. At 2 WAP, however, Pea1 had higher $\mathrm{NO}_{3}$ levels in water than any other treatment, similar to results in Expt. 1. No differences were observed in water $\mathrm{NH}_{4}$ at 2 WAP. At 4 WAP, Peal again had the highest levels of $\mathrm{NO}_{3}$ in soil water $(14.9 \mathrm{mg} / \mathrm{L})$ and was the only treatment with $\mathrm{NO}_{3}$ levels exceeding the recommended range in drinking water supplies $(10 \mathrm{mg} / \mathrm{L}$; EPA, 1972). Although Pea1 levels were high, levels were similar to Pea2 and CPL8. Again, no differences were observed in $\mathrm{NH}_{4}$ at 4 WAP. By 8 WAP, all $\mathrm{NO}_{3}$ levels were within acceptable ranges and all treatments had $\mathrm{NO}_{3}$ levels similar to the non-fertilized control, a trend that continued at $12 \mathrm{WAP}$. Ammonium levels at 8 WAP indicated no difference among any treatment; however, by 12 WAP, CPL8 again had very high $\mathrm{NH}_{4}$ levels exceeding recommended levels for drinking water. However, CPL2, CPL4, and CPL6 were similar.

Water $\mathrm{pH}$ and electrical conductivity. No differences were observed in water $\mathrm{pH}$ among any treatment until $8 \mathrm{WAP}$ when CPL8 $\mathrm{pH}$ was higher than CPL1 and the non-fertilized control (data not shown). At 12 WAP, water $\mathrm{pH}$ was highest in CPL4 (7.2), CPL8 (7.4), and CPL10 (7.1), whereas all other treatments had $\mathrm{pH}$ levels ranging from 6.8 to 7.0 (data not shown).

Table 6. Effect of fertilizers on nitrogen (presented as $\mathrm{mg} / \mathrm{L}$ of $\mathrm{NO}_{3}$ and $\mathrm{NH}_{4}$ ) in soil water, Expt. 2.

\begin{tabular}{|c|c|c|c|c|c|c|c|c|c|c|c|}
\hline \multirow[b]{2}{*}{ Treatment } & \multirow{2}{*}{$\begin{array}{c}\text { Rate } \\
\left(\mathrm{g} \mathrm{N} / \mathrm{m}^{2}\right)\end{array}$} & \multicolumn{2}{|c|}{$1 \mathrm{WAP}^{\mathrm{z}}$} & \multicolumn{2}{|c|}{$2 \mathrm{WAP}$} & \multicolumn{2}{|c|}{4 WAP } & \multicolumn{2}{|c|}{$8 \mathrm{WAP}$} & \multicolumn{2}{|c|}{12 WAP } \\
\hline & & $\mathrm{NO}_{3}$ & $\mathrm{NH}_{4}$ & $\mathrm{NO}_{3}$ & $\mathrm{NH}_{4}$ & $\mathrm{NO}_{3}$ & $\mathrm{NH}_{4}$ & $\mathrm{NO}_{3}$ & $\mathrm{NH}_{4}$ & $\mathrm{NO}_{3}$ & $\mathrm{NH}_{4}$ \\
\hline 13-13-13 Peafowl® & 4.9 & $2.7 a b^{y}$ & $1.3 \mathrm{ab}$ & $12.5 \mathrm{a}$ & $3.2 \mathrm{a}$ & $14.9 \mathrm{a}$ & $1.3 \mathrm{a}$ & $1.0 \mathrm{~b}$ & $1.1 \mathrm{a}$ & $1.0 \mathrm{a}$ & $1.6 \mathrm{~b}$ \\
\hline 13-13-13 Peafowl $®$ & 9.8 & $1.4 \mathrm{ab}$ & $0.97 \mathrm{ab}$ & $4.7 \mathrm{~b}$ & $4.1 \mathrm{a}$ & $8.4 \mathrm{ab}$ & $2.9 \mathrm{a}$ & $0.33 \mathrm{~b}$ & $0.57 \mathrm{a}$ & $1.1 \mathrm{a}$ & $0.0 \mathrm{~b}$ \\
\hline 13-13-13 Polyon® & 4.9 & $0.80 \mathrm{ab}$ & $1.6 \mathrm{a}$ & $0.43 \mathrm{~b}$ & $0.90 \mathrm{a}$ & $0.43 \mathrm{~b}$ & $1.4 \mathrm{a}$ & $1.2 \mathrm{~b}$ & $1.0 \mathrm{a}$ & $1.1 \mathrm{a}$ & $1.3 \mathrm{~b}$ \\
\hline 13-13-13 Polyon $\AA$ & 9.8 & $1.4 \mathrm{ab}$ & $1.1 \mathrm{ab}$ & $0.40 \mathrm{~b}$ & $0.53 \mathrm{a}$ & $0.50 \mathrm{~b}$ & $0.70 \mathrm{a}$ & $0.43 \mathrm{~b}$ & $0.67 \mathrm{a}$ & $0.87 \mathrm{a}$ & $0.40 \mathrm{~b}$ \\
\hline $\mathrm{CPL}^{\mathrm{x}}$ & 4.9 & $1.7 \mathrm{ab}$ & $0.83 \mathrm{ab}$ & $0.63 \mathrm{~b}$ & $0.47 \mathrm{a}$ & $0.53 \mathrm{~b}$ & $0.67 \mathrm{a}$ & $1.4 \mathrm{ab}$ & $1.3 \mathrm{a}$ & $0.73 \mathrm{a}$ & $0.67 \mathrm{~b}$ \\
\hline CPL & 9.8 & $0.10 \mathrm{~b}$ & $0.30 \mathrm{~b}$ & $0.53 \mathrm{~b}$ & $0.83 \mathrm{a}$ & $0.43 \mathrm{~b}$ & $1.0 \mathrm{a}$ & $1.5 \mathrm{ab}$ & $0.47 \mathrm{a}$ & $1.4 \mathrm{a}$ & $2.3 \mathrm{ab}$ \\
\hline CPL & 19.6 & $3.5 \mathrm{a}$ & $0.87 \mathrm{ab}$ & $0.87 \mathrm{~b}$ & $0.83 \mathrm{a}$ & $0.73 \mathrm{~b}$ & $1.8 \mathrm{a}$ & $1.8 \mathrm{ab}$ & $1.7 \mathrm{a}$ & $2.8 \mathrm{a}$ & $2.7 \mathrm{ab}$ \\
\hline $\mathrm{CPL}$ & 29.4 & $0.67 \mathrm{ab}$ & $0.93 \mathrm{ab}$ & $6.3 \mathrm{~b}$ & $1.0 \mathrm{a}$ & $0.70 \mathrm{~b}$ & $1.2 \mathrm{a}$ & $1.0 \mathrm{~b}$ & $2.0 \mathrm{a}$ & $3.3 \mathrm{a}$ & $2.1 \mathrm{ab}$ \\
\hline CPL & 39.2 & $0.97 \mathrm{ab}$ & $1.4 \mathrm{ab}$ & $5.3 \mathrm{~b}$ & $2.4 \mathrm{a}$ & $3.1 \mathrm{ab}$ & $3.6 \mathrm{a}$ & $6.4 \mathrm{a}$ & $1.1 \mathrm{a}$ & $0.83 \mathrm{a}$ & $10.3 \mathrm{a}$ \\
\hline CPL & 49 & $2.0 \mathrm{ab}$ & $1.2 \mathrm{ab}$ & $8.0 \mathrm{~b}$ & $1.9 \mathrm{a}$ & $0.90 \mathrm{~b}$ & $1.8 \mathrm{a}$ & $2.2 \mathrm{ab}$ & $0.47 \mathrm{a}$ & $0.90 \mathrm{a}$ & $0.13 \mathrm{~b}$ \\
\hline Non-fertilized control & $* * *$ & $2.4 \mathrm{ab}$ & $0.80 \mathrm{ab}$ & $0.53 \mathrm{~b}$ & $0.83 \mathrm{a}$ & $0.43 \mathrm{~b}$ & $1.2 \mathrm{a}$ & $3.4 \mathrm{ab}$ & $0.77 \mathrm{a}$ & $0.57 \mathrm{a}$ & $0.83 \mathrm{~b}$ \\
\hline
\end{tabular}

${ }^{\mathrm{z}} \mathrm{WAP}=$ Weeks after planting.

${ }^{y}$ Means within column followed by the same letter are not significantly different based on Duncan's multiple range test $(\alpha=0.05)$.

${ }^{\mathrm{x}} \mathrm{CPL}=$ Composted poultry litter. 
Water EC levels were similar among all treatments at $1 \mathrm{WAP}$. At $2 \mathrm{WAP}, \mathrm{CPL} 10$ had higher EC $\left(0.78 \mathrm{dS} \cdot \mathrm{m}^{-1}\right)$ than both rates of Polyon ( 0.23 and $0.25 \mathrm{dS} \cdot \mathrm{m}^{-1}$, respectively) as well as CPL1 and CPL2 (0.29 and 0.34 $\mathrm{dS} \cdot \mathrm{m}^{-1}$, respectively). At $12 \mathrm{WAP}, \mathrm{CPL} 8 \mathrm{had}$ slightly higher EC levels $\left(0.52 \mathrm{dS} \cdot \mathrm{m}^{-1}\right)$ than all other treatments with the exception of Pea1 $\left(0.39 \mathrm{dS} \cdot \mathrm{m}^{-1}\right)$ and CPL4 $\left(0.39 \mathrm{dS} \cdot \mathrm{m}^{-1}\right)$, which were similar.

\section{Discussion}

Similarities of plant growth and LCC in these studies indicate that use of CPL as a fertilizer for landscape annual bedding plants could produce plants equal in size and quality (LCC) to plants fertilized with traditional commercially available inorganic fertilizers. Final plant DW in both experiments indicates that petunias grew similarly when fertilized with CPL when compared with an inorganic fertilizer at the same rate. Verbena GI and DW in Expt. 1 also demonstrate that verbena grew similarly when fertilized with equal rates of CPL compared with inorganic fertilizers, and in Expt. 2, plants fertilized with CPL grew larger than when fertilized with inorganic fertilizers. In addition, few differences were noticed for LCC in either experiment, indicating that plants were not only similar in size, but also in quality. At the conclusion of each experiment, petunias had LCC equal to or higher to the inorganic treatments when the same rate of $\mathrm{N}$ was applied. Results were similar for verbena in Expt. 1; however, in Expt. 2, Poly2 had higher LCC than CPL2 or Pea2. Warmer temperatures at 12 WAP in Expt. 2 causing a release of Polyon fertilizer could have attributed to this difference; however, visual differences in foliar color were non-existent. As the rate of CPL increased, no differences were observed in terms of plant size or LCC. The reason for this is unclear; however, this may be because the highest rates of CPL provided more $\mathrm{N}$ and other nutrients than could be used by annual bedding plants, but no negative effects were observed.

Comparatively, a study by Altland et al. (2003) evaluated the growth of 'Peppermint Cooler' vinca [Catharanthus roseus (L.) G. Don 'Peppermint Cooler'], 'Bonanza Yellow' marigold (Tagetes patula L. 'Bonanza Yellow'), and 'Hawaii Blue' ageratum (Ageratum houstonianum Mill. 'Hawaii Blue') when fertilized with different fertilizer formulations, methods of application, and frequencies. Results indicated that organically based fertilizer resulted in larger, more attractive (higher foliar color ratings) plants than inorganic fertilizers.

Levels of $\mathrm{NO}_{3}$ and $\mathrm{NH}_{4}$ in leachates during Expt. 1 were extremely high in comparison with results from Expt. 2 and remained high in most treatments until $8 \mathrm{WAP}$. This is possibly the result of drought conditions for the initial portion of Expt. 1, which slowed N leaching, and more $\mathrm{N}$ was captured in the lysimeter during sample collection. Plant roots had likely not fully developed in early weeks of each experiment causing more $\mathrm{N}$ leaching than was seen in later weeks. In addition, GI of both species were considerably larger at 4 WAP during Expt. 2 (Table 4) than in Expt. 1 (Table 1), and it is likely that more nutrients were used by the plants during the early weeks of Expt. 2, causing less $\mathrm{N}$ leaching. In each experiment, $\mathrm{N}$ leaching $\left(\mathrm{NO}_{3}\right.$ and $\mathrm{NH}_{4}$ ) from CPL was less than or equal to $\mathrm{N}$ leaching compared with inorganic fertilizers at the same $\mathrm{N}$ rate.

In Expt. 1, $\mathrm{NO}_{3}$ and $\mathrm{NH}_{4}$ levels were consistently higher than recommended levels until 8 WAP. Although total N leaching was less in Expt. 2, especially in earlier weeks, $\mathrm{NO}_{3}$ and $\mathrm{NH}_{4}$ levels still exceeded recommended levels for drinking water $\left(10 \mathrm{mg} \cdot \mathrm{L}^{-1}\right)$ throughout both studies (EPA, 1972, 2009). A similar situation was reported by Timmons and Holt (1977) in which mean annual flowweighted concentration of inorganic $\mathrm{N}\left(\mathrm{NH}_{4}+\right.$ $\mathrm{NO}_{3}$ ) in surface runoff from unfertilized native prairie was $1.3 \mathrm{mg} \cdot \mathrm{L}^{-1}$ with a maximum value of $13.6 \mathrm{mg} \cdot \mathrm{L}^{-1}$ observed during a 5-year study. In addition, Sharpley et al. (1983) reported $\mathrm{NH}_{4}$ in runoff from unfertilized pasture $\left(0.9 \mathrm{mg} \cdot \mathrm{L}^{-1}\right)$ and $\mathrm{NO}_{3}$ levels consistently higher than the critical values associated with accelerated eutrophication $\left(0.3 \mathrm{mg} \cdot \mathrm{L}^{-1}\right)$. Consequently, Sharpley et al. (1983) reported that it appears to be unrealistic to attempt to attain or maintain $\mathrm{NH}_{4}$ or $\mathrm{NO}_{3}$ concentration in surface runoff from fertilized agricultural land below the recommended levels. Although in contrast this study examined $\mathrm{N}$ leaching through the soil profile, unfertilized plots often exceeded these critical ranges. Soil water samples would need to be collected at greater depths and closer to groundwater supplies to determine if the fertilization methods used in the current study would actually be detrimental to drinking water supplies.

A similar sequence of studies was conducted by Altland et al. (2002) in which organically based fertilizers (fresh cage layer poultry manure + recycled newspapers) were compared with commonly used inorganic fertilizer formulations. The organic fertilizer had higher total soil water $\mathrm{N}\left(\mathrm{NO}_{3}+\mathrm{NH}_{4}\right)$ in comparison with the inorganic formulations in the majority of experiments conducted. However, Altland's studies evaluated raw (uncomposted) poultry manure, whereas the current study used fully CPL. Although composting litter before application has been shown to reduce nutrient levels and value as an agricultural fertilizer (Tiquia and Tan, 2000), it has been shown to be beneficial in terms of reducing non-point source pollution from $\mathrm{N}$ leaching (Vervoort et al., 1998).

Results from these experiments demonstrate that CPL can be used successfully as a fertilizer for annual bedding plants. In most cases, plants grew as well or better when equal rates of fertilizer were applied either using CPL or commercial inorganic fertilizer formulations. With respect to non-point source pollution, these experiments also indicate that CPL could possibly be used at much higher rates of $\mathrm{N}$ than inorganic fertilizers while having similar levels of total $\mathrm{N}$ in soil solution. However, the highest rates of CPL applied in this study (CPL6, CPL8, and CPL10) would result in a gross overapplication of $\mathrm{N}$, far exceeding the needs of annual bedding plants. Consequently, overapplication of CPL would result in high amounts of other less used nutrients as well. One concern when using PL is $\mathrm{P}$ accumulation in soils as well as elevated levels of $\mathrm{P}$ in groundwater and surface runoff, leading to eutrophication (Moore et al., 1995). Field applications of PL at rates to meet plant $\mathrm{N}$ requirements normally result in overapplication of P. Phosphorus levels in soil or soil solution were not documented in these studies, and concerns over $\mathrm{P}$ accumulation in soil, surface runoff, or groundwater from repeated landscape applications of CPL will require further investigation.

As fertilizer prices continue to rise, landscapers and homeowners will begin to look for alternatives to expensive inorganic fertilizer sources. Composted poultry litter provides nutritional requirements needed for healthy plant growth, and use of CPL in the landscape provides poultry producers a new outlet for excess waste that is environmentally friendly. Composted poultry litter and other organic fertilizers do have disadvantages when compared with inorganic products in terms of nutrient-to-weight ratio, freight charges (depending on location), and the need for proper nutrient analysis before application. Composted poultry litter can however provide similar benefits to inorganic fertilizers in terms of plant growth and could supplement the need for other fertilizer sources while potentially minimizing negative environmental impacts.

\section{Literature Cited}

AAES. 2009. Economic impact of Alabama's green industry: Green industry growing. Spec. Rept. No. 7. Ala. Agri. Exp. Sta. Auburn University, AL.

Altland, J.E., C.H. Gilliam, J.H. Edwards, G.J. Keever, D.C. Fare, and J.L. Sibley. 2002 Fertilizer formulation and method of application influence bedding plant growth and nitrogen leaching in urban landscapes. J. Environ. Hort. 20:204-213.

Altland, J.E., C.H. Gilliam, J.H. Edwards, G.J. Keever, D.C. Fare, and J.L. Sibley. 2003. Fertilization methods affect growth, color and nitrogen leaching of winter annuals in landscape beds. J. Environ. Hort. 21:99-107.

Brown, D.G., K.M. Johnson, T.R. Loveland, and D.M. Theobald. 2005. Rural landuse trends in the conterminous United States, 1950-2000. Ecol. Appl. 15:1851-1863.

Burmester, C.H., C.W. Wood, and C.C. Mitchell. 1991. Broiler litter promising as nitrogen source for cotton. Highlights of Agr. Res. Auburn Univ. $38: 3$.

Dimitri, C. and C. Greene. 2002. Recent growth patterns in the U.S. organic foods market. Agr. Inform. Bul. No. 777. Economic Research Service, USDA

Environmental Protection Agency. 1972. Water quality criteria. U.S. Environmental Protection Agency, Washington, DC.

Environmental Protection Agency. 2009. Drinking water contaminants. 4 June 2009. <http://www. epa.gov/ogwdw000/contaminants/index.html $>$.

Feagley, S.E., W.J. Grichar, and J.D. Nerada. 2005. Use of chicken litter for bermuda grass production in south Texas. J. Sustain. Agr. 25: 67-90. 
Mills, H.A. and J.B. Jones. 1996. Plant analysis handbook II. MicroMacro Publishing, Athens, GA.

Mitchell, C.C., J.O. Donald, and J. Martin. 1995. The value and use of poultry waste as fertilizer. Ala. Coop. Ext. Sys. 3 July 2007. <http:// hubcap.clemson.edu/blpprt/Aub-44.html>.

Moore P.A., Jr., T.C. Daniel, A.N. Sharpley, and C.W. Wood. 1995. Poultry manure management: Environmentally sound options. J. Soil Water Conserv. 50:321-327.

Porch, D.W., C.H. Gilliam, J.E. Brown, J.L. Adrian, and J.T. Eason. 1990. Broiler litter vs commercial fertilizer produces earlier, larger, tomatoes. Highlights of Agr. Res. No. 37:5. Auburn University, AL.

Reeder, J.A., C.H. Gilliam, C.C. Mitchell, and J.O. Donald. 1992. Bedding plant response to incorporated broiler litter. Proc. Southern Nur. Assn. Res. Conf. Vol. 37:298-299.

Ribaudo, M. 2003. Managing manure: Clean water act regulations create imperative for livestock producers. 12 June 2011. < http://www.ers.usda. gov/amberwaves/Feb03/pdf/feature- managing\% 20manure.pdf $>$.

Sharpley, A.N., J.K. Syers, and R.W. Tillman 1983. Transport of ammonium and nitrate nitrogen in surface runoff from pasture as influenced by urea application. J. Water Air Soil Pollut. 20:425-430.

Sims, G.K., T.R. Ellsworth, and R.L. Mulvaney. 1995. Microscale determination of inorganic nitrogen in water and soil extracts. Commun. Soil Sci. Plant Anal. 26:303-316.

Soil Moisture Equipment Co. 2007. Operating instructions: 1900 soil water samplers. 1 Aug. 2007. <http://soilmoisture.com/pdf/1900.pdf $>$.

Tiquia, S.M. and N.F.Y. Tan. 2000. Fate of nitrogen during composing of chicken litter. Env. Polit. 110:535-541.

Timmons, D.R. and R.F. Holt. 1977. Nutrient losses in surface runoff from a native prairie. J. Environ. Qual. 6:369-373.
Vervoort, R.W., D.E. Radcliffe, M.L. Cabrera, and M. Latimore, Jr. 1998. Nutrient losses in surface and subsurface flow from pasture applied poultry litter and composted poultry litter. Nutr. Cycl. Agroecosyst. 50:287-290.

Warren, S.L. and C.D. Safley. 1990. Use of compost poultry litter in the horticulture industry. Proc. Natl. Waste Manage. Symp. Auburn University Printing Service. p. 144-149, Oct. 1990.

Wen-yuan, H. 2007. Tight supply and strong demand may raise U.S. nitrogen fertilizer prices. 24 Jan. 2008. <http://www.ers.usda.gov/Amber Waves/November07/Findings/TightSupply. htm>.

Williams, J.D. and C.C. Mitchell. 1997. Liming and fertilizing ornamental plants, Ala. Coop. Ext. Serv. Circ. 388.

Wolfe, K. and G. Humphries. 2007. Landscape and nursery utilization of composted manure. College. of Agr. and Environ. Sci. Rpt. 07-09. University of Georgia, Athen, GA. 